\title{
Preventing Metastasis by Targeting Lymphatic Vessels with Photodynamic Therapy Based on Nanostructured Photosensitizers
}

\section{Longo JPF ${ }^{1 *}$, MuehImann LA ${ }^{2}$, Almeida-Santos MFM ${ }^{1}$ and Azevedo RB ${ }^{1}$}

${ }^{1}$ Department of Genetics and Morphology, Institute of Biological Sciences, University of Brasilia, Brazil

${ }^{2}$ Faculty of Ceilandia, University of Brasilia, Brazil

Since the description of the Enhanced Permeability and Retention (EPR) related to the pathophysiology of tumor tissue [1], thousands reports have been published showing an improvement in the therapeutic index of classical chemotherapeutic drugs with the use of nanoparticles. The EPR effect is a phenomenon based on the presence of aberrant immature, tortuous, hyper permeable blood vessels, and a low density, dilated, leaky and discontinuous lymphatic vessels within tumor tissue [2]. This specific condition produce a space where some types of circulating materials can go in due to the high permeable vasculature; however the way out is not that easy. The key point of this phenomenon is the size cut-off natures of aberrant, enlarged, and discontinuous endothelium produced in tumor blood vessels. While normal constitutive vessels have intercellular openings with 1-100 $\mathrm{nm}$, tumor vessels have defective opening intercellular space with 400-500 $\mathrm{nm}$. These size differences produce a high permeable tissue environment to nanometric materials, fact that explain the efficacy improvement of drug when entrapped in drug nano-carriers [2].

As described previously, one of the most attractive features of EPR is the possibility of passively increasing nanoparticles input into tumor tissues. However, some publications have highlighted the possibility that nanoparticles can achieve not only the blood vasculature, but also the tumor draining lymphatic vessels. Lymphangiogenesis has been associated with inflammation, cancer metastasis, autoimmunity, tolerance and transplant rejection, and thus, targeted lymphatic ablation is a potential therapeutic strategy for treating or preventing such events [3].

As described above tumor lymphatic vessels are produced as aberrant enlarged forms that work as open roads to sentinel regional lymph nodes, which may pave the way for metastasis spread to distant organs, such as lung and liver. It's possible to use the EPR effect to reach these tumor-associated lymphatic vessels. In addition, an alternative choice to improve nanoparticle concentration within these structures is the intratumoral or peritumoral injection [4]. This strategy aims to place nanoparticles within the interstitial space, which are subsequently absorbed by draining lymphatic vessels due to a low pressure, a typical pattern of these vessels.

This approach was previous described [5] in an elegant experimental set-up. In that report, authors showed the specific ablation of tumor-associated lymphatic vessels, as well as destruction of in-transit metastatic tumor cells inside lymphatic vessels after peritumoral injection of photosensitizer (PS) associated to nanocarriers using Photodynamic Therapy (PDT). PDT is a combination of PS molecules and harmless light to produce an oxidative stress and cytotoxicity in the surrounding tissue where the PSs are accumulated [6]. Since lymphatic vessels passively accumulate the PS, PDT can be used as an innovative approach due to the specificity of the treatment. Noteworthy, this report showed that experimental animals presented significant reduction of distant metastasis when treated with this PDT protocol [5].

After this first publication describing the ablation of lymphatic vessels with PDT, we published two reports involving the prevention and reduction of metastasis with PDT and nanostructured photosensitizers in two different tumor models: (1) a mice tongue tumor [7], showing the prevention of loco-regional lymph node metastasis; and a mice breast cancer model [8], showing the prevention of lung metastasis after PDT application.

This lymphatic vessels ablation after PDT is strongly related to the basic mechanisms involved in PDT. Basically, PDT works due to three different biological mechanisms: (1) first the photochemical reaction acts directly against target cells, inducing a direct cytotoxic activity [9]; (2) second, PDT promotes the shutdown of surrounding blood and lymphatic vessels [10]; and (3) third, PDT stimulate immune system against tumor cells. It is worth to note that some authors [3] demonstrated that it is possible to establish PDT conditions to specifically destroy lymphatic vessels.

Thus, concerning the second mechanism, related to the antivascular therapy, we propose that PDT could be applied to destroy not only blood vessels structures, but also lymphatic tumor vessels,

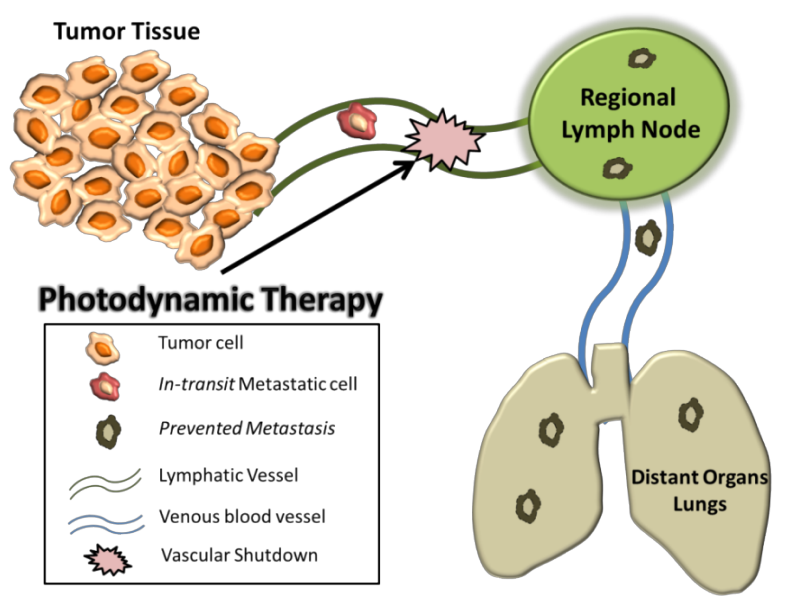

Figure 1: General pathway of metastatic spread and the PDT prevention activity.

*Corresponding author: Longo JPF, Department of Genetics and Morphology, Institute of Biological Sciences, University of Brasília, Brazil, Tel: 5561 3107-3087; E-mail: jplongo82@gmail.com

Received June 27, 2015; Accepted July 30, 2015; Published October 01, 2015

Citation: Longo JPF, Muehlmann LA, Almeida-Santos MFM, Azevedo RB (2015) Preventing Metastasis by Targeting Lymphatic Vessels with Photodynamic Therapy Based on Nanostructured Photosensitizers. J Nanomed Nanotechnol 6: 318. doi:10.4172/2157-7439.1000318

Copyright: @ 2015 Longo JPF, et al. This is an open-access article distributed under the terms of the Creative Commons Attribution License, which permits unrestricted use, distribution, and reproduction in any medium, provided the original author and source are credited. 
Citation: Longo JPF, Muehlmann LA, Almeida-Santos MFM, Azevedo RB (2015) Preventing Metastasis by Targeting Lymphatic Vessels with Photodynamic Therapy Based on Nanostructured Photosensitizers. J Nanomed Nanotechnol 6: 318. doi:10.4172/2157-7439.1000318

Page 2 of 2

therefore preventing metastatic cell migration by this pathway. A scheme describing the general pathway of metastatic spread and the PDT prevention activity is presented in Figure 1. The idea described previously [5] and confirmed by our group $[7,8]$ is that PDT can be used as vascular shutdown tool to destroy those cell migration pathways, preventing, at least partially the establishment of metastatic lesions. It is also important to call the attention that besides blood vessels shutdown PDT can also stimulate the immune system, making it a useful protocol to be applied in combination to other cancer therapy, in order to improve treatment outcomes and patient survival after the diagnosis with neoplastic lesions (Figure 1).

\section{References}

1. Matsumura $Y$, Maeda $H$ (1986) A new concept for macromolecular therapeutics in cancer chemotherapy: mechanism of tumoritropic accumulation of proteins and the antitumor agent smancs. Cancer research 46: 6387-6392.

2. Torchilin V (2011) Tumor delivery of macromolecular drugs based on the EPR effect. Advanced drug delivery reviews 63: 131-135.

3. Kilarski WW, Muchowicz A, Wachowska M, Mezyk-Kopec R, Golab J, et al. (2014) Optimization and regeneration kinetics of lymphatic-specific photodynamic therapy in the mouse dermis. Angiogenesis 17: 347-357.
4. Torchilin VP (2005) Recent advances with liposomes as pharmaceutical carriers. Nat Rev Drug Discov 4: 145-160.

5. Tammela T, Saaristo A, Holopainen A, Yla-Herttuala S, Andersson LC, et al (2011) Photodynamic ablation of lymphatic vessels and intralymphatic cancer cells prevents metastasis. Sci transl med 3: 69ra11.

6. MuehImann LA, Joanitti GA, Silva JR, Longo JP, Azevedo RB (2011) Liposomal photosensitizers: potential platforms for anticancer photodynamic therapy. Braz J Med Biol Res 44: 729-737.

7. Bicalho LS, Longo JP, Cavalcanti CE, Simioni AR, Bocca AL, et al. (2013) Photodynamic therapy leads to complete remission of tongue tumors and inhibits metastases to regional lymph nodes. J Biomed Nanotechnol 9: 811 818.

8. Longo JPF (2016) Prevention of Distant Lung Metastasis after Photodynamic Therapy Application in a Breast Cancer Tumor Model. Journal of Biomedical Nanotechnology.

9. Muehlmann LA, Ma BC, Longo JP, Almeida Santos MF, Azevedo RB (2014) A luminum-phthalocyanine chloride associated to poly(methyl vinyl ether-comaleic anhydride) nanoparticles as a new third-generation photosensitizer for anticancer photodynamic therapy. Int J Nanomedicine 9: 1199-1213.

10. Mijan MC, Longo JPF, Melo LND, Simioni AR, Tedesco AC, (2014) Vascular shutdown and pro-inflammatory cytokine expression in breast cancer tumors after photodynamic therapy mediated by nano-sized liposomes containing aluminium-chloride-phthalocyanine. J Nanomed Nanotechnol 5: 1-9. 